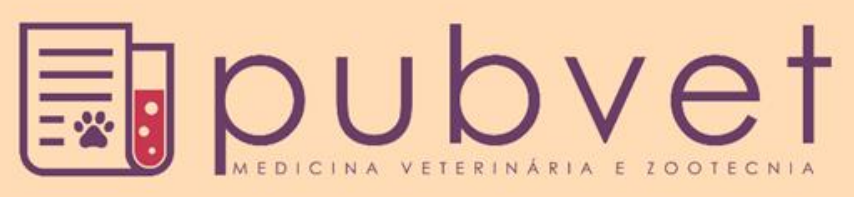

ISSN $1982-1263$

https://doi.org/10.31533/pubvet.v13n6a355.1-7

\title{
Descrição morfométrica da anatomia cardíaca canina e humana: proximidades e distanciamentos
}

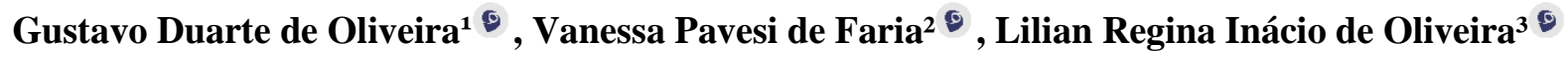 \\ ${ }^{I}$ Especialista/UEG. Pesquisador CAPES/UEG - GPEFDL. Graduando em Medicina Veterinária pela Universidade Montes Belos \\ ${ }^{2}$ Mestre, Bolsista Fapesp pelo Departamento de Patologia Animal da Faculdade de Ciências Agrárias e Veterinárias - \\ FCAV/Unesp, Jaboticabal. Docente do curso de Bacharelado em Medicina Veterinária da Universidade Montes Belos \\ ${ }^{3}$ Especialista em Tecnologias Aplicadas ao Ensino de Biologia pela Universidade Federal de Goiás, Brasil
}

Resumo. Artigo desenvolvido como primeiro resultado da pesquisa oriunda do Projeto de Estudos em Cardiologia no Centro Universitário Montes Belos - UniMB, curso de Medicina Veterinária - Estudo Comparativo do Perfil Cardiológico entre Cães e Homens: parâmetros das principais cardiopatias e similaridades anatômicas. O objetivo específico desta análise é reavaliar as proximidades entre as classes hominidae e canidae quanto ao quesito anatômico do coração. As proximidades, semelhanças e os distanciamentos, diferenças entre a morfometria cardíaca do cão e do humano. O referenciamento teórico teve como base argumentativa de Ghoshal (1986), Carvalho et al. (2002) e Queiroz et al. (2018) para suporte argumentativo na discussão morfométrica cardíaca canina e humana, Cunningham (2011) para a fisiologia cardíaca e outros autores, de igual importância para a sustentação do arquétipo argumentativo deste trabalho.

Palavras-chave: Anatomia, coração, morfologia

\section{Morphometric description of canine and human heart anatomy: proximities and distances}

\begin{abstract}
Article developed as a result of research Project of Studies in Cardiology at the University Center Montes Belos - UniMB, Veterinary Medicine course - Comparative Study of the Cardiological Profile between Dogs and Men: parameters of the main cardiopathies and anatomical similarities. The specific objective of this analysis is to reassess the proximities between the classes' hominidae and canidae regarding the anatomical aspect of the heart. The proximities, similarities and distances, differences between the cardiac morphometric of the dog and the human. The theoretical referencing was based on an argumentative basis by Ghoshal (1986), Carvalho et al. (2002) and Queiroz et al. (2018) for argumentative support in cardiac and human cardiac morphometric discussion, Cunningham (2011) for cardiac physiology and other authors with equal importance for the support of the argumentative archetype of this work.
\end{abstract}

Keywords: Anatomy, heart, morphology

\section{Descripción morfométrica de la anatomía cardiaca canina y humana: cercanías y distanciamientos}

Resumen. Este artículo se desarrolló como el primer resultado de la investigación del Proyecto de Estudios de Cardiología en el Centro Universitario Montes Belos - UniMB, Medicina Veterinaria - Estudio Comparativo del Perfil Cardiológico entre Perros y Hombres: parámetros de las principales cardiopatías y similitudes anatómicas. El objetivo 
específico de este análisis es reevaluar las proximidades entre las clases hominidae y canidae con respecto al aspecto anatómico del corazón. Las proximidades, similitudes y distancias, diferencias entre la morfometría cardíaca del perro y el humano. La referencia teórica se basó en Ghoshal (1986), Carvalho et al. (2002) e Queiroz et al. (2018) para el apoyo argumentativo en la discusión sobre la morfometría cardíaca canina y humana, Cunningham (2011) para la fisiología cardíaca y otros autores, de igual importancia para el soporte del arquetipo argumentativo de este trabajo.

Palabras clave: anatomía, corazón, morfología

\section{Introdução}

O coração é o órgão central do sistema circulatório (König \& Liebich, 2011). O coração é um órgão muscular, responsável por carrear sangue para irrigar tecidos do organismo com $\mathrm{O}_{2} \mathrm{e}$, por isso, é conhecido como a bomba muscular do aparelho circulatório (Carvalho et al., 2002; Carvalho et al., 1986). Tal bomba muscular é também reconhecida como câmara muscular e está subdividido em quatro cavidades que exercem função de contração em ritmos sequenciados e regulares cuja finalidade é direcionar sangue para os vasos sanguíneos (Nusshag \& Romero, 1980). Numa descrição anatômica, o coração é delimitado por dois ventrículos principais (Ventriculi cordis) posicionados no lado esquerdo e direito. Cada um desses ventrículos é precedido por uma porção cavitária atrial, átrio (atrium cordis), totalizando as quatro cavidades de sua composição (König \& Liebich, 2011).

O coração porta o sangue em suas cavidades com o objetivo de assegurar que haja quantidade suficiente para preencher cada átrio e cada ventrículo. Se a habilidade de portar e direcionar o sangue em suas cavidades e espaçamentos for comprometido, é sinal de desenvolvimento de algum quadro patológico. As cavidades são separadas por válvulas, as quatro válvulas são: as semilunares direita e esquerda, a bicúspide e a tricúspide. A parede muscular que se compõe o coração é constituída de três camadas, a epicárdica, a miocárdica e a endocárdica (Zachary et al., 2012). O coração é constituído por um tipo específico de músculo que é o Músculo Estriado Cardíaco (MEC). O MEC é morfologicamente composto por filamentos de actina e miosina (Cunningham, 2011). O que difere o MEC do músculo esquelético é a membrana que delimita a célula, conhecida como disco. O disco possui uma resistência elétrica que facilita com que o potencial de movimento exercido pelo Sistema Nervoso Central seja efetivado sem desgaste ou rompimento tecidual com recorrência de necrose. De acordo com Matsuo et al. (1988), os átrios são morfologicamente reconhecidos por serem delgados e seus ventrículos mais espessos, sendo que a sua parede ventricular esquerda é três vezes, aproximadamente, maior que a parede ventricular direita, e, por questões fisiológicas ou patológicas como a sobrecarga da pulsação circulatória, pode aumentar ainda mais por ação compensatória.

Uma curiosidade que Cunningham (2011) explora é que, até o século XVII acreditava-se que, o sangue percorria pelo organismo como um sistema de maré. O coração fluía para fora do coração e adentrava os vasos e o mesmo sangue retornava ao coração pelo mesmo fluxo inverso. Foi somente no século seguinte que se descobriu a realidade do sistema circulatório, e o termo circulatório que condiz conceitualmente com o movimento de percorrer um caminho numa única via de forma unidirecional, e não um "vai e volta", mas somente uma "ida".

O sistema circulatório o qual possui o coração como motor e propulsor central abastece os tecidos dos demais órgãos com substratos metabólicos: $\mathrm{O}_{2}$, ácidos graxos, glicose; mensageiros químicos essenciais como hormônios, insulina e retira para excreção os produtos metabólicos como ácido lático e $\mathrm{CO}_{2}$.

\section{Objetivo}

Este artigo se propõe a apresentar e discutir as características gerais e específicas da anatomia cardíaca dos caninos e humanos. O termo "aproximações" diz respeito às semelhanças, portanto, o que aproxima as espécies; e "distanciamento", as diferenças, ou seja, o que os torna distantes um do outro no quesito anatômico e fisiológico. 


\section{Material e métodos}

A metodologia usada neste estudo descritivo argumentativo foi fundamentada numa revisão bibliográfica, a nível qualitativo, portanto, uma série de descrições conceituais, desenvolvido com base nas leituras direcionadas e orientadas. Pesquisas claras e objetivamente expostas para o meio acadêmico. As leituras e fichamentos das publicações para este artigo foram escritos a partir das análises e cobertura de dados dos pesquisadores presentes no referenciamento bibliográfico.

Uma reunião de informações acerca da morfologia cardíaca humana e canina com base nas publicações disponíveis em plataformas oficiais de pesquisa online credenciadas e reconhecidas pelos órgãos cabíveis à pesquisa e extensão tecnológica. Ghoshal (1986), Carvalho et al. (2002) e Queiroz et al. (2018) para suporte argumentativo na discussão morfométrica cardíaca canina e humana, Cunningham (2011) para a fisiologia cardíaca e outros autores, de igual importância para a sustentação do arquétipo argumentativo deste trabalho.

\section{Resultados e discussão}

Os cães e humanos descendem da mesma linha de superclasses: agnatas, gnatostomatas e tetrápodes (Höfling, 1995). Analisando a superclasse tetrápode, os animais a ela pertencentes são quadrúpedes terrestres subdivididos em quatro subclasses: os répteis, animais terrestres exclusivos que introduzem ovos amnióticos; as aves, organismos aéreos que desenvolveram penas pelo processo histórico de evolução; os anfíbios, seres terrestres e aquáticos; e os mamíferos, animais com o corpo coberto de pelos e que se alimentam de leite materno no início da vida (Höfling, 1995; Romer \& Parsons, 1985).

Seres vertebrados e mandibulados, os cães e humanos possuem o coração como uma estrutura única, ventral, anteriorizada e alinhada ao seu eixo longitudinal (Fishman \& Chien, 1997; Romer \& Parsons, 1985) sua localização se dá no tronco do animal (Romer \& Parsons, 1985). O estudo está argumentado em proximidades e distanciamentos da morfometria cardíaca de ambas as espécies. O coração dos seres da família canidae possuem a mesma morfologia e a mesma localização em relação ao corpo que os seres da família hominidae, pois todos são mamíferos. Nos mamíferos, é anatomicamente dividido em quatro câmaras, sendo dois átrios e dois ventrículos (Queiroz et al., 2018). Apresenta aspecto ovoide, com ápice rombo e arredondado (Carvalho et al., 2002). Semelhante à anatomia humana, o ápice sendo uma estrutura mais externa do coração e angulada em formato pontiagudo formando uma base para o coração repousar sobre o diafragma. O coração humano também possui formato ovoide irregular (Sobotta, 2002). O peso do coração de um humano é de aproximadamente 250 gramas e pode variar para 400 gramas. A simetria anatômica do corpo corresponde o tamanho do coração de um homem adulto semelhante ao tamanho da mão do mesmo homem fechada, um seja, um punho.

$\mathrm{O}$ peso do coração de um cão de tamanho médio é de aproximadamente $40 \mathrm{~g}$ e pode chegar aos $600 \mathrm{~g}$, correspondendo respectivamente de 0,9 a 2,2\% do peso corporal. Entretanto, o peso é uma entidade relativa, portanto está sujeito a uma ampla variação, sendo maior em cães de caça, são treinados para desenvolver a habilidade da velocidade ou de suportar trabalho por longos períodos. Já em caninos com sobrepeso e de hábitos sedentários, o peso do coração pode representar apenas 0,5\% do peso corporal (Ghoshal, 1986).

\section{Morfometria}

A palavra morfometria possui sua etimologia aplicada ao grego, e ao segmentar em dois radicais para extrair o entendimento correspondente a que a palavra designa, serão encontrados os termos - morphé (forma) e - metrikós (métrica, medida). Dentro do léxico da área médica veterinária, o uso da palavra morfometria é o estudo da forma e das medidas correspondente à anatomia aplicada.

Esse método tem por objetivo, tornar mais fácil e preciso a coleta, a apresentação e a análise dos resultados obtidos na pesquisa e laboratórios. Permitindo ainda relacionar as diferentes estruturas anatômicas com as suas funções.

\section{Aproximações morfométricas}

Assim como na espécie humana, o coração canino não se diferencia quanto ao sexo. Portanto, as diferenças morfométricas cardíacas não são aplicadas quanto ao gênero, ou seja, o sexo não é determinante para diferir morfologicamente e diferenciá-lo como um coração de macho (cão, homem) 
ou fêmea (cadela, mulher) dentro da própria espécie (Bienvenu \& Drolet, 1991). Ou um diferencial entre a espécie canina e humana.

A morfologia cardíaca possui as mesmas estruturas cavitárias correspondentes aos átrios e ventrículos. A mesma disposição muscular: músculo atrial, músculo ventricular e as fibras musculares excitatórias e condutoras (Junqueira \& Carneiro, 2013).

É percebida a mesma nomenclatura anatômica: base do coração (local de conexão com os principais vasos sanguíneos como aorta, artérias e veias pulmonares), ápice do coração (conformidade aguda que direciona o coração centralizado ao mediastino estar voltado para o lado esquerdo).

Numa análise externa para uma descrição anatômica, a parte brilhosa que contempla toda a estrutura do coração é chamada de Pericárdio Fibroso. Presente também em corações humanos, cuja função é estabilizar e proteger o coração como uma camada de revestimento contra movimentos não indicados e de força abrupta, caso o indivíduo/animal corra e pule. O coração está exposto para visão dorsal, visão cabível para facilitar o registro da medida do eixo cardíaco vertical. Entretanto, mesmo assim nessa posição, é possível observar o ápice do coração na sua extremidade medida na régua na margem de 27 cm e a base do coração, medida na régua na margem dos 36/37 cm (Figura 1 e 2).

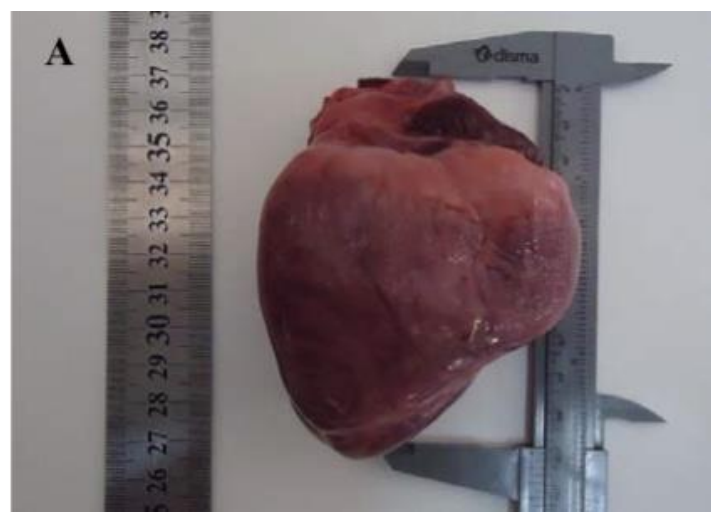

Figura 1. Coração canino. Medida do eixo cardíaco vertical. Fonte: Queiroz et al. (2018).

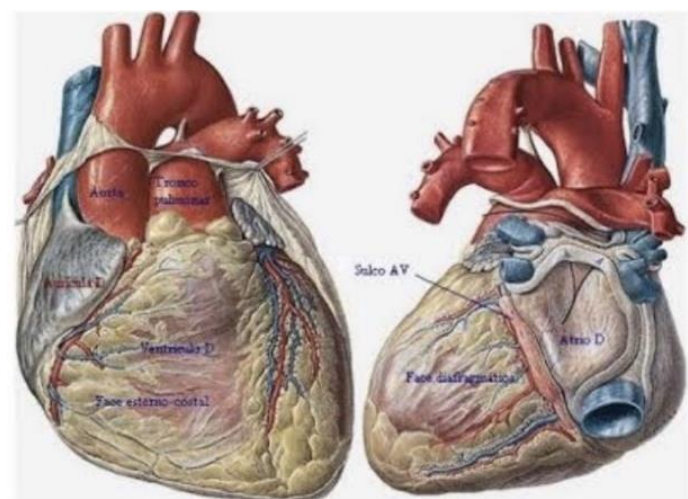

Figura 2. Coração humano. Exposição frontal e dorsal do coração. Disponível por Sobotta (2002).

A base do coração é o local onde concentram os principais vasos sanguíneos para realização da macro circulação (sangue já oxigenado pelos pulmões, ao serem direcionados pela artéria aorta é encaminhado para todo o corpo) e a microcirculação (sangue que chega pela veia cava superior e é destinado para as veias e artérias pulmonares para oxigenação nos pulmões). Na figura 3 e 4 é evidente a exposição das cavidades musculares atriais e ventriculares. As cordas tendíneas e os músculos papilares. O Septo cardíaco e as válvulas bicúspide e tricúspide. As cordas tendíneas são tendões que possuem a função de ligar cada valva cardíaca aos músculos pectíneos do coração para realização da passagem sanguínea de uma câmara para outra. Músculos papilares são encontrados nos ventrículos direito e esquerdos e são à base de ligação das cordas tendíneas que em conjunto, realizam a função de permitir ou não a passagem de sangue para a câmara muscular cardíaca seguinte.

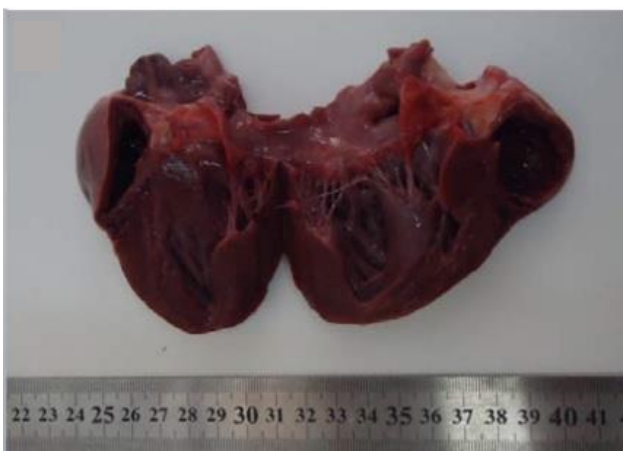

Figura 3. Coração canino. Abertura longitudinal e exposição das câmaras cardíacas. Fonte: Queiroz et al. (2018).

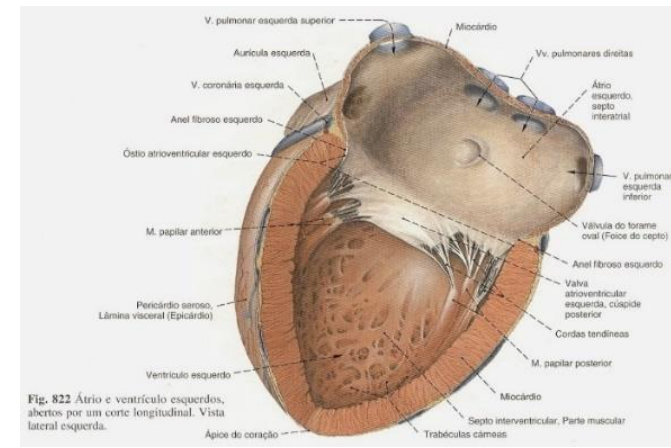

Figura 4. Coração humano. Exposição frontal atrial e ventricular esquerdo. Fonte: Sobotta (2002). 
Septo cardíaco ou também conhecido como septo interventricular, é uma parede muscular que divide e diferencia o ventrículo esquerdo do ventrículo direito, o que impede o sangue venoso misturar com o sangue arterial.

\section{Distanciamentos morfométricos}

A disposição dos vasos na base do coração é o que diferencia o que distancia a anatomia do coração canino em relação do coração humano. A Veia Cava superior em humanos possui sua localização paralela superior esquerda a Artéria Aorta, o que resulta no projeto do contorno do coração na parede torácica anterior (Sobotta, 2002).

Portanto, em humanos, a veia cava superior está acima do átrio direito. A veia cava inferior segue a mesma linha longitudinal da Veia Cava Superior e está envolvido pelo seio da veia cava. A veia cava inferior pode ser bem analisada numa visão dorsal do coração (Figura 5 e 6). Por consequência, é percebida uma canalização sequencial da veia cava em humanos envolvidos por um seio.

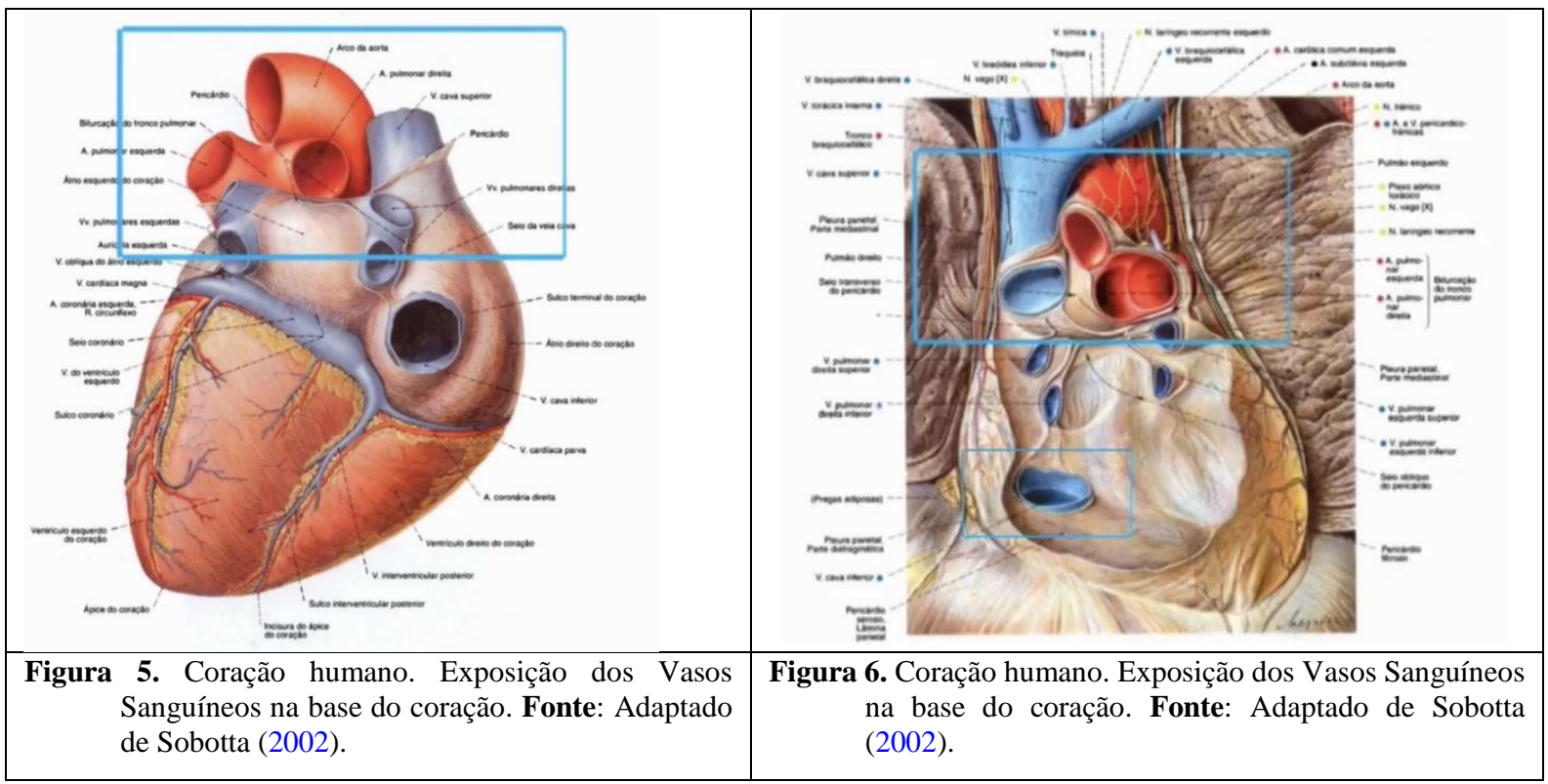

$\mathrm{Na}$ imagem acima destacada, é possível visualizar a veia cava superior e a veia cava inferior separadas pelo seio da veia cava. O coração está exposto numa visão dorsal, o ápice direcionado para o lado direito.

A disposição da veia cava em cães é diferente da anatomia humana. O distanciamento já é observado na nomenclatura, pois na morfometria canina, as veias cavas mudam seu nome para veia cava cranial e veia cava caudal. A sua disposição no órgão e também em relação ao corpo do animal, faz com que sua distribuição seja latitudinal ao coração e não longitudinal.

As veias cavas craniais e caudais possuem uma sequência de exposição de visão paralela ao órgão principal, que é o coração, como é possível visualizar na figura 7. Outro distanciamento é a não formação de arco pela artéria aorta. Em humanos, a artéria aorta forma um arco aórtico que dele saem o canal tronco braquioencefálico, artéria carótida comum e a artéria subclávia. Ao contrário, no coração canino não ocorre à formação do arco aórtico. A artéria aorta segue uma canalização contínua e dela saem os seguimentos artéria subclávia esquerda e tronco braquiocefálico. Como não há o arco aórtico para encobrir a bifurcação da artéria pulmonar, no coração canino, somente a artéria pulmonar direita toca a artéria aórtica.

No coração canino, as veias pulmonares estão localizadas na região superior do átrio esquerdo disponibilizados em locais paralelos, portanto, as quatro veias, locadas em pares estão expostas no átrio esquerdo como é visto na figura 7.

No coração humano, as veias pulmonares são designadas como veia pulmonar superior e inferior, o que condiz com uma posição hierárquica de uma veia acima da outra. Ou seja, temos as veias 
pulmonares superiores e inferiores esquerdas e veias pulmonares superior e inferior direitas, como visto na figura 5

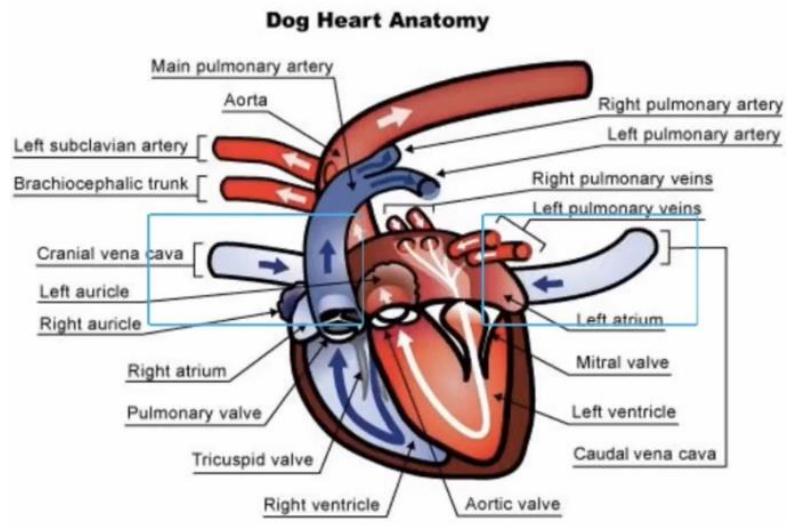

Figura 7. Coração canino. Exposição dos Vasos Sanguíneos na base do coração. Fonte: Banco de Imagens: Anatomia II da Universidade Montes Belos

\section{Considerações finais}

A cardiologia veterinária está crescendo significativamente em pesquisa e extensão nas últimas décadas. Os animais domésticos por desfrutarem de uma qualidade de vida melhor com o passar dos anos conquistaram longevidade de vida e assim, estão propícios ao desenvolvimento de patologias cardiovasculares.

As cardiomiopatias e as tipologias de más formações congênitas cardíacas entre cães e humanos são semelhantes e desenvolvem com mesmo quadro clínico. Este estudo veio provar as semelhanças e diferenças, ou melhor, aproximações e distanciamentos entre a estrutura anatômica do coração canino e humano.

Foi detectado pelas leituras que, as cavidades musculares são semelhantes e as unidades musculares também. São quatro câmaras: dois átrios e dois ventrículos revestidos por musculo estriado cardíaco com fibras musculares excitatórias e condutoras.

Os distanciamentos se dão pela disposição dos principais vasos sanguíneos na base do coração. A exposição da veia cava, artéria aorta, veias e artérias pulmonares são distintos quanto a locação no coração, variando em morfologia latitudinal (canino) e longitudinal (humano).

A variação que se apresenta dos vasos entre os espécimes em análise se dá primeiramente pela posição segundo os planos e eixos de cada organismo. A análise dos planos e eixos é diferente do cão para o homem, devido a sua forma anatômica, o cão é quadrúpede e o homem é bípede.

A locação dos vasos sanguíneos na base do coração e do posicionamento cardíaco mediante a cavidade cardiotorácica é diferente em um ser que se locomove de forma ereta com dois membros inferiores, no caso humano as pernas e com quatro membros: membros torácicos e pélvicos, no cão. Por este motivo temos a não formação do arco aórtico, e a disposição paralela das veias pulmonares no átrio esquerdo que facilita o contato com os pulmões e outras peculiaridades que distanciam a anatomia cardíaca canina da humana.

\section{Referências bibliográficas}

Bienvenu, J. G. \& Drolet, R. (1991). A quantitative study of cardiac ventricular mass in dogs. Canadian Journal of Veterinary Research, 55(4):305-309.

Carvalho, L. M. M., Sousa, A. L., Andrade, P. H. E., Guerra, P. C. \& Alves, F. R. (2002). Morfometria cardíaca externa em cães adultos. Revista Pesquisa em Foco, 10(2):47-51. 
Carvalho, R. G., Krichenko, A., Giublin, P., Varela, A., Amorin, M. J., Bueno, R. L., . . Loures, D. R. R. (1986). Transplante heterotópico do coraçäo sem auxílio da circulaçäo extracorpórea: estudo experimental em cäes. Revista Brasileira de Cirúrgia Cardiovascular, 1(2):49-56.

Cunningham, J. (2011). Tratado de fisiologia veterinária. Rio de Janeiro: Guanabara Koogan.

Fishman, M. C. \& Chien, K. R. (1997). Fashioning the vertebrate heart: earliest embryonic decisions. Development, 124(11):2099-2117.

Ghoshal, N. G. (1986). Coração e artérias do carnívoro. Anatomia dos animais domésticos, 51497-1550.

Höfling, E. (1995). Chordata: manual para um curso prático. São Paulo, Brasil: Edusp.

Junqueira, L. C. \& Carneiro, J. C. (2013). Histologia Básica (12 ed. ed.). Rio de Janeiro: Guanabara Koogan.

König, H. E. \& Liebich, H.-G. (2011). Anatomia dos Animais Domésticos: Artmed Editora.

Matsuo, T., Carabello, B. A., Nagatomo, Y., Koide, M., Hamawaki, M., Zile, R. \& McDermott, P. J. (1988). Mechanisms of cardiac hypertrophy in canine volume overload. American Journal of Physiology, 275(1):66-74.

Nusshag, W. M. d. A. \& Romero, J. (1980). Compendio de anatomía y fisiología de los animales domésticos. Zaragoza: Acribia.

Queiroz, L. L., Moura, L. R. \& Moura, V. M. B. D. (2018). Morphometric assessment of canine heart without macroscopial visible changes caused by cardiac disease. Ciência Animal Brasileira, $19 \mathrm{e} 43748$.

Romer, A. S. \& Parsons, T. S. (1985). Anatomia comparada dos vertebrados. São Paulo, Brasil: Atheneu.

Sobotta, J. (2002). Atlas de anatomia humana (Vol. 2). Rio de Janeiro, Brasil: Guanabara Koogan.

Zachary, J. F., McGavin, D. \& McGavin, M. D. (2012). Bases da patologia em veterinária. Rio de Janeiro: Elsevier Brasil.

Recebido: 2 de maio, 2019.

Aprovado: 29 de maio, 2019.

Publicado: 4 de julho, 2019.

Licenciamento: Este artigo é publicado na modalidade Acesso Aberto sob a licença Creative Commons Atribuição 4.0 (CCBY 4.0), a qual permite uso irrestrito, distribuição, reprodução em qualquer meio, desde que o autor e a fonte sejam devidamente creditados. 